\title{
Data mining-based detection of the clinical effect on motion style acupuncture therapy combined with conventional acupuncture therapy in chronic neck pain
}

\author{
Ruo-yun Lyu ${ }^{\mathrm{a}, \mathrm{c}}$, Zong-lin Wen ${ }^{\mathrm{a}, \mathrm{b}}$, Wen-chao Tang ${ }^{\mathrm{a}}$, Xu-ming Yang ${ }^{\mathrm{a}}$, Jun-ling Wen ${ }^{\mathrm{a}}$, \\ Bo Wang ${ }^{\mathrm{b}, *}$ and Ming $\mathrm{Gao}^{\mathrm{a}, *}$ \\ ${ }^{a}$ School of Acupuncture-Moxibustion and Tuina, Shanghai University of Traditional Chinese Medicine, \\ Shanghai, China \\ ${ }^{\mathrm{b}}$ Shuguang Hospital Affiliated to Shanghai University of Traditional Chinese Medicine, Shanghai, China \\ 'Shanghai Tenth People's Hospital, Shanghai, China
}

\begin{abstract}
.
BACKGROUND: Neck pain is the most common symptom of cervical spondylosis.

OBJECTIVE: To discuss the indications for conventional acupuncture therapy (CAT) and motion style acupuncture therapy (MSAT) combined with CAT in neck pain patients using data mining.

METHOD: Seventy-six participants with neck pain were recruited and randomly divided into two paralleled groups. Participants in intervention group received two-course MSAT at Houxi (SI3) and CAT at local neck region, while the control group received CAT at neck only. Take the SF-36 score scale and pain pressure threshold (PPT) values as the raw data and use data-mining to evaluate the clinical effect of two treatments.

RESULT: Both groups have the best effect after one-time and one-course treatment. The main factors influencing effect most are Role-Emotional (RE), Physical Functioning (PF) and Social Functioning (SF) in intervention group, while it transfers to General Health $(\mathrm{GH}), \mathrm{RE}$ and SF in control group. To patients in intervention group who scores 41.70 to 68.70 in PF before treatment, MSAT can effectively improve the restriction of activities and play an analgesic effect; to patients in control group who score 56.09 to 66.09 in GH before treatment, CAT may have curative effect.

CONCLUSION: Both MSAT and CAT can improve the life-quality of neck pain patients. Patients with high general health status before treatment can choose CAT, while patients who score mild to moderate decline in physiological function can choose MSAT combined with CAT.
\end{abstract}

Keywords: Chronic neck pain, acupuncture, motion style acupuncture therapy, SF-36, pain pressure threshold

\section{Introduction}

Cervical spondylosis $[1,2]$ is a series of syndromes caused by chronic strain or acute injury and may

${ }^{*}$ Corresponding authors: Bo Wang, Shanghai Shuguang Hospital Affiliated to Shanghai University of Traditional Chinese Medicine, Shanghai 201203, China. E-mail: carloswb@ 163.com and Ming Gao, School of Acupuncture-Moxibustion and Tuina, Shanghai University of Traditional Chinese Medicine, Postbox 146\#, 1200 Cailun Road, Pudong New Area, Shanghai 201203, China. Tel.: +86 21 51322450; Fax.: +86 21 51322264; E-mail: gaoming688@ sina.com. 
manifest as neck pain, stiffness, dizziness, numbness in arms and weakness in legs. As the most common symptom of cervical spondylosis, neck pain (NP) was ranked the fourth leading cause of disabilityadjusted life years (DALYs) globally in 2017 [3,4], and the global incidence of neck pain was calculated to be $16.2 \%$ in 2018 [5]. Although the conventional acupuncture therapy (CAT) is widely used in neck pain treatment for the obvious instant effect, studies [6,7] show that the recurrence rate of neck pain keeps high due to the poor maintenance effect of CAT. On the other hand, several studies [8-10] considered that CAT combined with motion style acupuncture therapy (MSAT) has better efficacy, especially when pain is caused by the skeletal motor system. Therefore, both treatments are widely applied in the clinic.

However, whether the treatment is suitable for the patient should be taken into consideration. The application of CAT and MSAT has not been standardized yet. Most of the acupuncturists choose the treatment by clinical symptom and medical history, which leads to the huge difference in clinical effect.

In fact, there are many methods to evaluate the severity of neck pain, and many of them can be quantified, among which we choose the pain pressure threshold (PPT) and the MOS item short from health survey (SF-36) as reference to discuss the relationship between them and the clinical effect.

To reduce statistical bias, we take the further data mining method on the basis of traditional statistics. In brief, this study aims to discuss the indications for CAT and MSAT combined with CAT in neck pain patients through data mining method, so that the result can give advice to clinical treatment for different kind of neck pain patients.

\section{Methods}

\subsection{Trial design}

The trial is designed as an assessor-blinded RCT to confirm whether the effect of CAT combined with MSAT is better than CAT. Seventy-six participants with chronic neck pain will be randomly assigned into intervention group and control group with the ratio of 1:1. The process of the trial is detailed in Fig. 1 and Table 1.

This trial is designed in accordance with the Declaration of Helsinki and has been approved by the ethics committee of Shanghai Shuguang Hospital (Permission number: 2018-580-09-01). The trial has been registered at the Chinese Clinical Trial Registry (no. ChiCTR1900025413). Informed consent form of all participants will be obtained before enrollment after informing them about the outline and detail of the trial.

\subsection{Recruitment}

Patients with chronic neck pain were recruited from Acupuncture and Moxibustion Department of Shanghai Shuguang Hospital. The main recruitment method was advertising on social media, such as WeChat and Weibo, which is similar to Twitter and WhatsApp. A diagnosis will be made by orthopedists after patients consent to participate the study, followed with a baseline evaluation performed by research assistant.

\subsubsection{Inclusion criteria}

1) Diagnostic criteria for cervical spondylosis; 2) Men or women aged between 20-55 years; 3) Chronic neck pain for at least 3 months; 4) Current visual analogue scale (VAS) score between 3 and 7; 5) No painkillers or glucocorticoids in the past 2 weeks; 6) Willing to sign the informed consent form and participate into clinical trial. 
Table 1

Time to visit and data collection

\begin{tabular}{|c|c|c|c|c|c|}
\hline \multirow[b]{3}{*}{ Time node } & \multicolumn{3}{|c|}{ Study period } & \multirow[b]{2}{*}{ Visit 4} & \multirow[b]{2}{*}{ Visit 5} \\
\hline & Visit 1 & Visit 2 & Visit 3 & & \\
\hline & $-7 \sim 0$ day & $\begin{array}{l}\text { One-time } \\
\text { treatment }\end{array}$ & $\begin{array}{c}\text { One-course } \\
\text { treatment }\end{array}$ & $\begin{array}{c}\text { Two-course } \\
\text { treatment }\end{array}$ & $\begin{array}{l}\text { One-month } \\
\text { follow-up }\end{array}$ \\
\hline Informed consent & $\bullet$ & & & & \\
\hline Randomization & $\bullet$ & & & & \\
\hline General information & - & & & & \\
\hline Medical history & $\bullet$ & & & & \\
\hline Imaging examination & $\bullet$ & & & & \\
\hline Assessment & - & & & & \\
\hline PE & $\bullet$ & $\bullet$ & $\bullet$ & $\bullet$ & $\bullet$ \\
\hline PPT & $\bullet$ & $\bullet$ & $\bullet$ & $\bullet$ & $\bullet$ \\
\hline SF-36 & $\bullet$ & & & $\bullet$ & $\bullet$ \\
\hline Safety observation & & $\bullet$ & $\bullet$ & $\bullet$ & $\bullet$ \\
\hline Adverse event record & & $\bullet$ & $\bullet$ & $\bullet$ & $\bullet$ \\
\hline Obedience evaluation & & - & - & - & - \\
\hline Combined treatment records & & $\bullet$ & $\bullet$ & $\bullet$ & • \\
\hline
\end{tabular}

*If the subjects are directly enrolled at the first visit, i.e., visit 1 and visit 2 events coincide, visit 2 index will be taken as the baseline. ${ }^{*} \mathrm{PE}$, physical examination; PPT, pain pressure threshold; SF-36, the MOS item short from health survey.

\subsubsection{Exclusion criteria}

1) Pregnant or lactating women; 2) Diagnosis as cervical spondylotic myelopathy, or other indications for absolute surgery; 3 ) History with serious diseases such as cardiovascular, cerebrovascular, liver, kidney, hematopoietic, digestive system, or mental illness; 4) Having received relevant treatments that may influence the effect of the study; 5) Unable to understand and fill out the scales related to the research; 6) Reluctant to join the trial.

\subsection{Interventions}

The treatments were taken in Acupuncture and Moxibustion Department of Shanghai Shuguang Hospital by acupuncturists with more than 5 years clinical experience. In order to minimize the environment bias and emotion effect, all treatments were provided at afternoon in the same consulting room.

\subsubsection{Control group: CAT}

The control group accepted CAT at local acupoints of neck according to textbook named Acupuncture and Moxibustion [11], including Cervical Jiaji (EXB2), Fengchi (GB20), Tianzhu (BL10), Jianjin (GB21), Tianzong (SI11) and Ashi points (Table 2 and Fig. 2). The treatment took twice a week for 5 weeks with a total of 2 courses. The single-use stainless steel needle $(0.25 \times 40 \mathrm{~mm})$ (Suzhou Medical Products Factory Co., Ltd. Suzhou 215005, China) was used and Deqi sensation (a sensation of sour, numbness or distension) was achieved during process. Needle was retained for 20 mins and then withdrew quickly. Patients were required to exercise their neck in appropriate range after treatment.

\subsubsection{Intervention group: CAT combined with MSAT}

The treatment interval and times of intervention group were the same as that of the control group. In addition to CAT same as the control group, MSAT at Houxi (SI3) of painful side was further applied. Patients were required to exercise their neck up and down, left and right slowly during needling, while the range of exercise was up to the tolerate of pain. The whole process of MSAT was sustained for 5 times exercise cycle. Sustained-release or prophylactic analgesics were not allowed. 


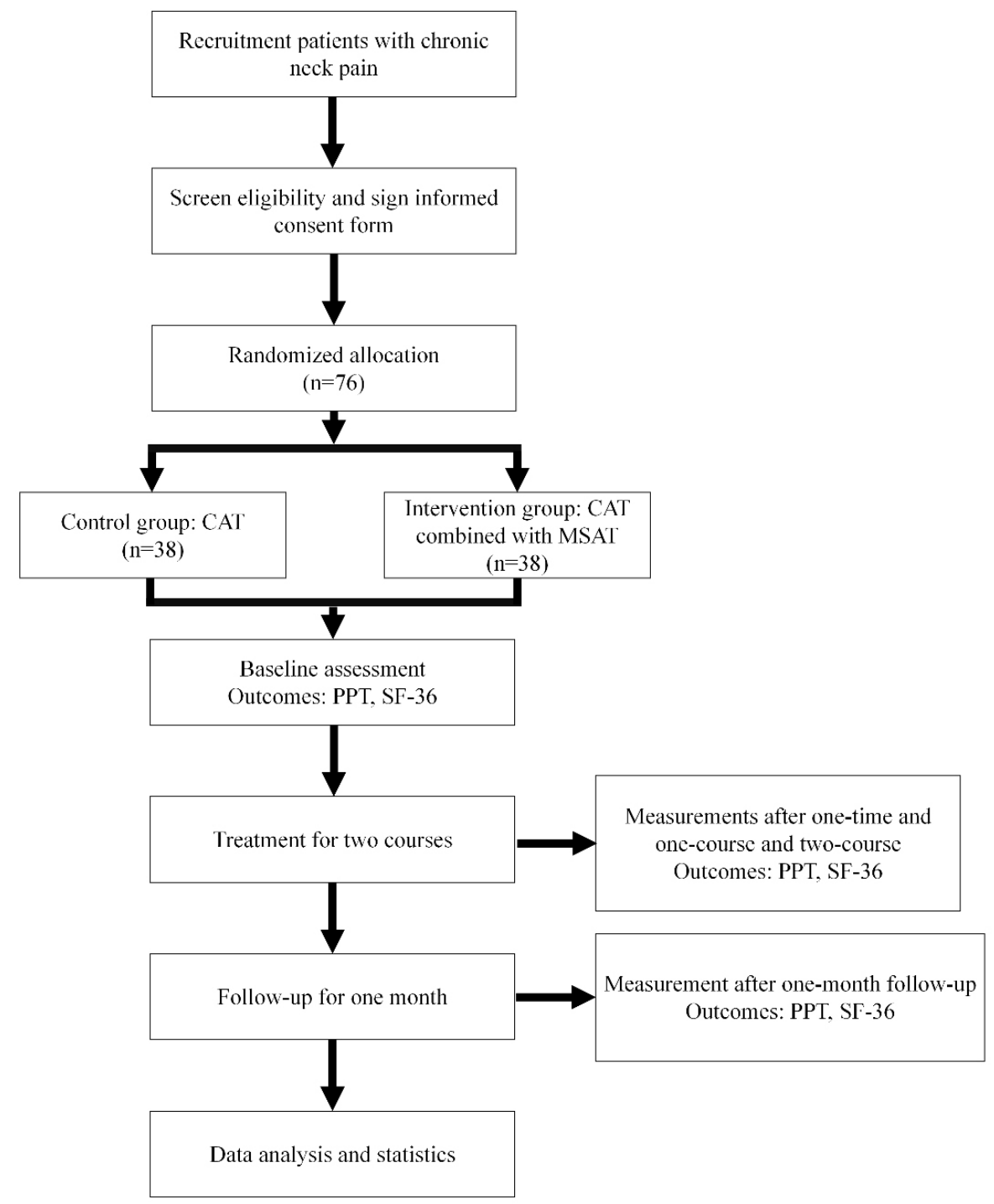

Fig. 1. Flowchart of the study.

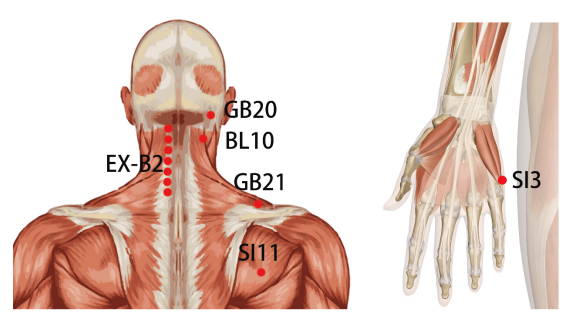

Fig. 2. Location of acupoints. 
Table 2

Acupoint locations and standards used for locating acupoints used in this trial*

\begin{tabular}{|c|c|c|c|}
\hline Acupoints & Locations & For treatment & For measurement \\
\hline GB20s & $\begin{array}{l}\text { In the anterior region of the neck, inferior to the occipital } \\
\text { bone, in the depression between the origins of } \\
\text { sternocleidomastoid and the trapezius muscles. }\end{array}$ & $\sqrt{ }$ & \\
\hline BL10s & $\begin{array}{l}\text { In the posterior region of the neck, at the same level of the } \\
\text { superior border of the spinous process of the second cervical } \\
\text { vertebra }(\mathrm{C} 2) \text {, in the depression lateral to the trapezius } \\
\text { muscles. }\end{array}$ & $\sqrt{ }$ & \\
\hline $\mathrm{EX}-\mathrm{B} 2 \mathrm{~s}$ & $\begin{array}{l}\text { In the posterior region of the neck, } 0.5 \mathrm{~B} \text {-cun bilateral to the } \\
\text { posterior median line, under the spinous process, between } \\
\text { the first cervical vertebrae and the seventh cervical vertebrae. }\end{array}$ & $\begin{array}{l}\text { The second, fourth and } \\
\text { sixth cervical vertebrae } \\
\text { level of both sides. }\end{array}$ & $\begin{array}{l}\text { The fourth and sixth } \\
\text { cervical vertebrae } \\
\text { level of both sides. }\end{array}$ \\
\hline SI11s & $\begin{array}{l}\text { In the scapular region, in the depression between the upper } \\
\text { one third and the lower two thirds of the line connecting the } \\
\text { midpoint of the spine of the scapula with the inferior angle } \\
\text { of the scapula. }\end{array}$ & $\sqrt{ }$ & \\
\hline GB21s & $\begin{array}{l}\text { In the posterior region of the neck, at the midpoint of the line } \\
\text { connecting the spinous process of the seventh cervical } \\
\text { vertebra (C7) with the lateral end of the acromion. }\end{array}$ & $\sqrt{ }$ & \\
\hline SI3s & $\begin{array}{l}\text { On the dorsum of the hand, in the depression proximal to the } \\
\text { ulnar side of the fifth metacarpophalangeal joint, at the } \\
\text { border between the red and white flesh. }\end{array}$ & $\sqrt{ }$ & \\
\hline
\end{tabular}

${ }^{*}$ Reference: WHO standard acupuncture point locations in the Western Pacific Region 2008. B-cun, proportional bone (skeletal) cun.

\subsection{Randomization and blinding}

The randomized program is developed using Statistical Package for the Social Sciences (SPSS) Ver.23.0 (IBM, New York, USA), and the department nurses will use the opaque random envelope to achieve the allocation concealment. The random envelope is marked with the serial number of the group, and group number is printed in the envelope. The envelopes are sequentially opened according to the time sequence of the included cases, and the control group and the intervention group are divided according to the groups in the envelope.

The participants and the acupuncturist will not be blinded because of specialization of acupuncture manipulation. However, the data analysis and efficacy assessment will be performed by the research assistant and blinded in order to decrease analysis bias.

\subsection{Outcomes}

\subsubsection{Pain pressure threshold}

PPT measurement has been widely used in relatively objective pain intensity qualification [12,13]. Four fixed points (Fig. 3), including EX-B2s at the fourth and the sixth cervical vertebra on both sides, have been selected for measurement, and the value of pressure at each point will be shown clearly on the digital screen of measuring apparatus (Force One FDIX Multi-capacity Digital Force Gage).

\subsubsection{Quality of life}

SF-36 [14] is a health-related questionnaire in eight dimensions, including Role-Emotional (RE), Role-Physical (RP), Physical Functioning (PF), Social Functioning (SF), General Health (GH), Bodily Pain (BP), Vitality (VT) and Mental Health (MH), which may provide a more comprehensive reference for patients' life quality. 


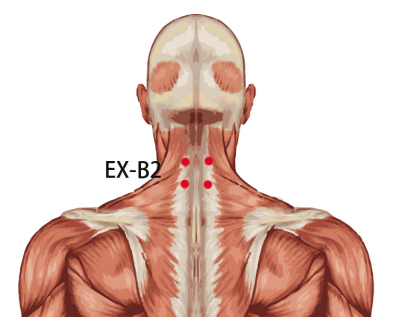

Fig. 3. Location of measurement points.

\section{Analysis}

\subsection{Statistical analyses}

The principle of Intention-To-Treat (ITT) analysis and Per-Protocol (PP) analysis will be followed in the process of statistical analysis. The Last Observation Carried Forward Analysis (LOCF) rule will be applied for missing data in ITT analysis. All the statistical analysis will be performed using SPSS ver. 23.0 with a significance level of 0.05 .

Two-sample $t$-test for quantitative data or Chi-square test for qualitative data is performed as homogeneity test, and the covariance analysis will also be performed if an adjustment is needed for a baseline characteristic. The analyses is focused on whether statistically better treatment outcomes could be achieved in the intervention group. $P$ values less than 0.05 are considered statistically significant and tests are 2-sided.

Two-sample $T$-test or Wilcoxon rank-sum test will be used for the comparison of the two groups and to determine differences according to normality. The mean change as well as interaction between groups and observed time of PPT at five-time nodes will be analysis using two-factor repeated measure analysis.

\subsection{Data mining algorithm}

The gray system theory proposes the concept of gray relational grade analyses on ach subsystem, and is used to investigate the numerical correlation between the various subsystems (or factors) in a certain way. Therefore, gray correlation analyses (GRA) provide a quantitative measure for the development of a system change trend, and are suitable for dynamic process analyses [15]. Information entropy is a measure of the amount of information needed to eliminate uncertainty, that is, the amount of information that an unknown event may contain. It includes Renyi entropy, Tsallis entropy, Shannon entropy and other forms. At present, analysis methods based on information entropy have been deepened in many scientific fields. Shannon entropy is a measurement method that characterizes information, which can quantify information with a specific distribution, and mainly focuses on the uncertainty of information [16]. As a measure of correlation analysis, mutual information is essentially the relative entropy of the product of the joint distribution of two random variables and their independent distributions. The biggest advantage is that it can effectively describe the nonlinear relationship between variables [17]. Association knowledge is knowledge that reflects the dependence or connection between an event and others. The important feature of association rules is "natural combination", which is very useful for discovering patterns in subsets of all attributes [18].

\subsubsection{GRA in acupuncture effect of different time points}

Use the GRA to compare the changes of the PPT values of $C 4_{L}, C 4_{R}, C 6_{L}$, and $C 6_{R}$ and analyze the 
Table 3

Baseline before treatment

\begin{tabular}{llllc}
\hline \multicolumn{2}{l}{ Male, $n(\%)$} & Intervention group $(n=38)$ & Control group $(n=38)$ & $P$ \\
Age & & $27.00(25.00,34.00)$ & 8 & 0.77 \\
PPT & $C 4_{L}$ & $30.54 \pm 7.59$ & $26.00(24.25,41.50)$ & 0.88 \\
& $C 4_{R}$ & $30.17 \pm 5.85$ & $26.46 \pm 6.66$ & 0.25 \\
& $C 6_{L}$ & $34.69 \pm 8.31$ & $27.30 \pm 8.05$ & 0.36 \\
& $C 6_{R}$ & $36.23 \pm 8.41$ & $31.75 \pm 6.71$ & 0.32 \\
SF-36 & PF & $90.00(75.00,100.00)$ & $85.00 \pm 6.62$ & 0.12 \\
& RP & $75.00(25.00,100.00)$ & $75.00(25.00,100.00)$ & 0.71 \\
& BP & $62.00(41.00,74.00)$ & $62.00(62.00,74.00)$ & 0.15 \\
& GH & $58.70 \pm 21.52$ & $58.76 \pm 24.56$ & 0.84 \\
& VT & $70.00(60.00,80.00)$ & $65.00(50.00,73.75)$ & 0.24 \\
& SF & $80.00(70.00,90.00)$ & $80.00(70.00,90.00)$ & 0.92 \\
RE & $75.00(50.00,100.00)$ & $87.50(50.00,100.00)$ & 0.76 \\
MH & $68.00(56.00,76.00)$ & $68.00(60.00,76.00)$ & 0.69 \\
\hline
\end{tabular}

${ }^{*}(\mathrm{x} \pm \mathrm{S})$ was used to represent the result of data in normal distribution; $\mathrm{M}\left(Q_{L} ; Q_{U}\right)$ was used to represent the result of data in non-normal distribution.

curative effect characteristics of different acupuncture treatments at five specified time nodes, including baseline before treatment, after one-time, one-course, two-course treatment and one-month follow-up.

\subsubsection{Qualitative analysis in acupuncture effect by mutual information method based on Shannon entropy}

The mutual information method based on Shannon entropy was used to analyze the correlation between the changes in PPT after treatment and SF-36 before treatment, which can characterize the nonlinear relationship between the two, and also discover the certain health assessment items that play an important role in the effect of acupuncture treatment [17].

\subsubsection{Quantitative exploration in acupuncture effect by association algorithm}

In this study, an association algorithm was used to discover the hidden correlation between the changes of PPT before and after treatment and the main health status of the two groups of patients before treatment.

\section{Result}

\subsection{Baseline}

A total of seventy-six participants with neck pain were recruited for this study, and five participants fell off ( 3 of intervention group and 2 of control group). The shedding rate was $6.6 \%$.

In baseline calculation, there were no significant differences in gender, average age, PPT and SF-36 before treatment between control group and intervention group $(P<0.05)$ (Table 3$)$.

\subsection{GRA in Acupuncture effect of different time points}

GRA showed that every acupoint had different characteristic in both group at four time nodes (Table 4). Take $C 4_{L}$ as example. In intervention group, the effect was better after one-time treatment (correlation of 0.68 ), then the correlation got smaller after one-course treatment (correlation of 0.10 ) and the improvement got slower after two-course treatment (correlation of 0.14), however, the correlation gradually increased 
Table 4

Correlation analysis between clinical effect and time nodes on four acupints in both group

\begin{tabular}{llcccc}
\hline & & $\begin{array}{c}\text { One-time } \\
\text { treatment }\end{array}$ & $\begin{array}{c}\text { One-course } \\
\text { treatment }\end{array}$ & $\begin{array}{c}\text { Two-course } \\
\text { treatment }\end{array}$ & $\begin{array}{c}\text { One-month } \\
\text { follow-up }\end{array}$ \\
\hline$C 4_{L}$ & Control group & 0.48 & 0.55 & 0.46 & 0.26 \\
& Interventional group & 0.68 & 0.10 & 0.14 & 0.17 \\
$C 4_{R}$ & Control group & 0.60 & 0.40 & 0.24 & 0.20 \\
& Interventional group & 0.61 & 0.40 & 0.43 & 0.40 \\
$C 6_{L}$ & Control group & 0.57 & 0.28 & 0.17 & 0.40 \\
& Interventional group & 0.67 & 0.71 & 0.34 & 0.55 \\
$C 6_{R}$ & Control group & 0.76 & 0.40 & 0.45 & 0.21 \\
& Interventional group & 0.76 & 0.12 & 0.10 & 0.40 \\
\hline
\end{tabular}

\section{Correlation of PPT at C4}

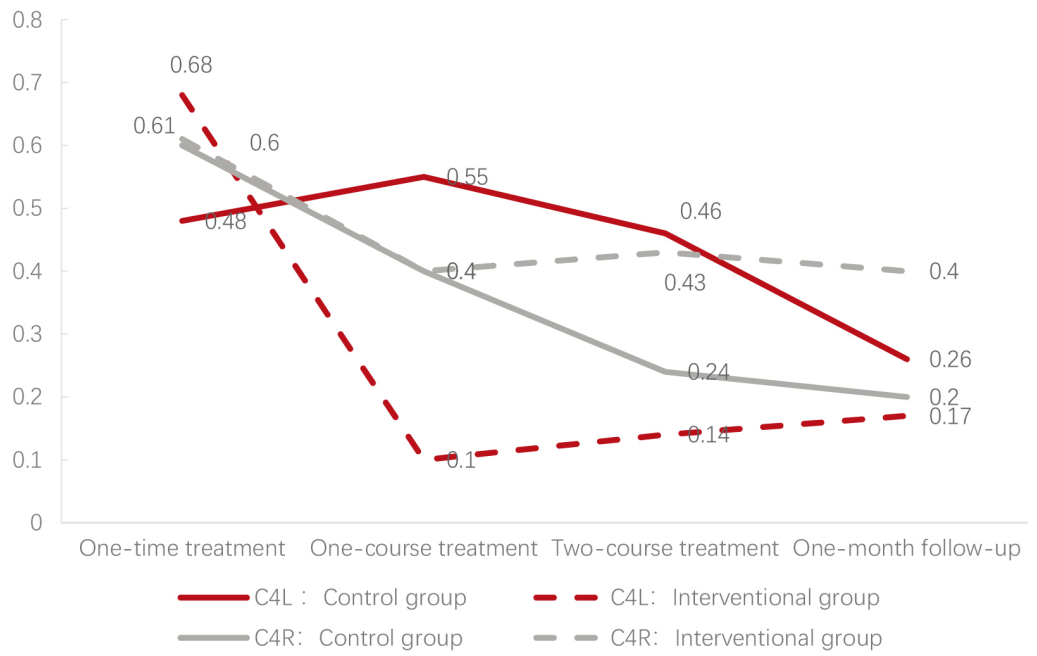

Fig. 4. Correlation of PPT at C4.

after one-month follow-up (correlation of 0.18). In control group, the effect was general after one-time treatment (correlation of 0.44), while it improved after one-course treatment (correlation of 0.55) and the correlation gradually got smaller after two-course treatment and one-month follow-up (correlation of 0.55 and 0.26). The correlation of other acupoints are shown in Figs 4 and 5.

Through the results of data mining in intervention group, the GRA shows that as the instant effect of acupuncture is the basement of long-term effect, the best effect appears after one-time treatment in $C 4_{L}, C 4_{R}$ and $C 6_{L}$, while it appears after one-course treatment in $C 6_{R}$. In control group, the best effect appears after one-course treatment in $C 4_{L}$, and appears after one-time treatment in another three acupoints. After one-month follow-up, the relational coefficient of 4 acupoints are similar or higher than that after two-course treatment in intervention group, while in control group only the relational coefficient of $C 6_{L}$ is higher than that after two-course treatment, the other three acupoints all show downward trend.

\subsection{The influence of Health status before treatment on PPT by mutual information method based on Shannon entropy}

Mining the correlation between SF-36 and PPT changes of $C 4_{L}, C 4_{R}, C 6_{L}, C 6_{R}$ before and after treatment by mutual information method based on Shannon entropy can describe the non-liner relationship 
Table 5

Correlation analysis of Shannon entropy between SF-36 and PPT before treatment

\begin{tabular}{llcccccccc}
\hline & & PF & RP & BP & GH & VT & SF & RE & MH \\
\hline Rank of correlation & Intervention group & 0.03 & 0.02 & 0.01 & 0.00 & 0.01 & 0.02 & 0.03 & 0.00 \\
& & 2 & 4 & 6 & 7 & 5 & 3 & 1 & 8 \\
& Control group & 0.00 & 0.00 & 0.00 & 0.03 & 0.00 & 0.01 & 0.01 & 0.00 \\
& & 6 & 8 & 7 & 1 & 4 & 3 & 2 & 5 \\
\hline
\end{tabular}

\section{Correlation of PPT at C6}

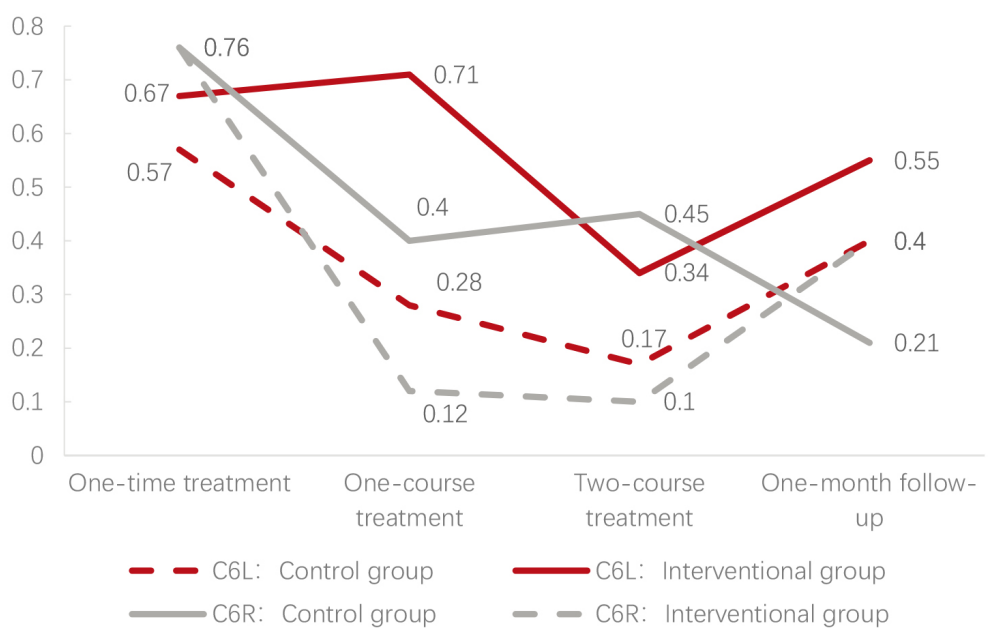

Fig. 5. Correlation of PPT at C6.

between them and certain the health assessment items before treatment that play an important role in the effect of acupuncture treatment [19].

Set the eight items in SF-36 as X, the PPT changes of $C 4_{L}, C 4_{R}, C 6_{L}, C 6_{R}$ before and after treatment as Y. The relationship between them is shown in Table 5.

The result showed that the "correlation coefficients" of the relevant items in the SF-36 and the PPT of four acupoints in the intervention group are RE, PF, SF, RP, VT, BP, GH, MH (ranked from strong to weak), which meant the main influencing factors of intervention group are RE, PF and SF, while those in control group are $\mathrm{GH}, \mathrm{RE}$ and $\mathrm{SF}$, which means the difference of main influencing factors between intervention group and control group is $\mathrm{PF}$ and GH. Therefore, MSAT combined with CAT is more objective to improve PF, and CAT is more universal to improve $\mathrm{GH}$.

\subsection{Quantitative mining the correlation of GH before treatment and acupuncture effect by association algorithm}

\subsubsection{Implicit correlation between GH and clinical effect in control group}

Set the difference of PPT before and after treatment in control group as input variable, GH as predictor variable. Use association algorithm to mine, generate dozens of rules. When the probability is 1.00 and the importance is 0.94 , the six strongest rules are generated:

\section{Probability Importance Rule}

(1) $1.000 .94 C 4_{R}<0.09, C 4_{L}=-3.6-5.56->\mathrm{GH}=56.09-66.09$. 
(2) $1.000 .94 C 4_{R}<0.09, C 6_{L}=0.63-10.73->\mathrm{GH}=56.09-66.09$.

(3) $1.000 .94 C 6_{R}=11-14, C 4_{L}=-3.60-5.56->\mathrm{GH}=56.09-66.09$.

(4) 1.00 0.94 $C 6_{R}=11.00-14.00, C 6_{R}=0.63-10.73->\mathrm{GH}=56.09-66.09$.

(5) $1.000 .94 C 4_{R}<0.09->\mathrm{GH}=56.09-66.09$.

(6) $1.000 .94 C 4_{R}<0.09, C 6_{L}=11.00-14.00->\mathrm{GH}=56.09-66.09$

The result showed that no matter how PPT changed, it was correlated with " $\mathrm{GH}=56.0862-66.0948$ ", which meant the pain improvement in control group by CAT was related to the variety of GH scored among 56.09 to 66.09. In another word, patient who scored among 56.09 to 66.09 in GH before treatment, could be better treated by CAT.

\subsubsection{Implicit correlation between $P F$ and clinical effect in intervention group}

Set the difference of PPT before and after treatment in intervention group as input variable, PF as predictor variable. Use association algorithm to mine, generate dozens of rules. When the probability is 1.00 and the importance is 0.76 , the six strongest rules are generated:

\section{Probability Importance Rule}

(1) 1.00 0.76: $C_{L}>=12.32, C 4_{R}>=13.59->\mathrm{PF}=41.70-68.70$.

(2) 1.00 0.76: $C 4_{L}>=12.32, C 6_{R}=12.78-18.09->\mathrm{PF}=41.70-68.70$.

(3) 1.00 0.76: $C 4_{R}<-1.95, C 4_{L}=1.75-7.11->\mathrm{PF}=41.70-68.70$.

(4) 1.00 0.76: $C 6_{L}>=19.51, C 4_{R}>=13.59->\mathrm{PF}=41.70-68.70$.

(5) 1.00 0.76: $C 6_{L}>=19.51, C 6_{R}=12.78-18.09->\mathrm{PF}=41.70-68.70$.

(6) 1.00 0.76: $C 4_{L}>=12.32, C 6_{L}>=19.51->\mathrm{PF}=41.70-68.70$.

The result showed that no matter how the four acupoints made up, "PF $=41.70-68.70$ " improved, which meant the improvement of PF in intervention group was correlated to the score among 41.70 to 68.70 before treatment. In another word, MSAT combined with CAT can better ameliorate pain in patient who scored among 41.70 to 68.70 in PF before treatment.

\section{Discussion}

In recent years, the incidence of cervical spondylosis continuously rises for the popularity of electronic products and the maintenance of long-term forward neck posture [20,21]. In this study, by measuring the raw data of PPT and SF-36 before and after MSAT combined with CAT and CAT treatment, data mining technology is used to deeply explore the implicit correlation between the two and different acupuncture treatments, as well as their clinical effects. The inherent regularity characteristics of the data and symptoms provide technical support for in-depth exploration of the clinical efficacy of different acupuncture treatments of neck pain.

SF and RE are the common main factors in both groups that influence PPT. RE refers to the limitation of function due to own emotional problems. SF represents the impact of physiological and psychological problems on the quantity and quality of social activities and is an indicator to evaluate the effect of health on social activities [14]. Neck pain belongs to deep body pain and usually causes by the damage of soft tissue and muscle. When deep pain occurs, the skeletal muscle near the painful region contracts reflectively and leads to local tissue ischemia, which further aggravates the pain [22]. Patients with body pain may have negative explanations for pain, which in turn may lead to pain physiology, cognition and fear avoidance reactions [23]. Fear and negative perception of pain may lead to escape, cause disability and painful movement, and form a negative pain cycle [24]. In severe cases, it can further affect the 
patient's psychological and social activities [25]. The results shows that the analgesic effect of acupuncture in the treatment of neck pain has greatly improved the patient's emotional function and social function. If the patient feels less pain and gains more mobility through encouragement and acupuncture therapy, a positive cycle can be created and the treatment effect can be improved [26]. Modern research [27-29] believes that acupuncture mainly exerts analgesic effects by inhibiting the pain transmission pathway or regulating the release of cytokines, and due to its remarkable and time-dependent analgesic effect, the analgesic effect of acupuncture has been widely used in treatment of various diseases.

In addition to the common factors, PF and GH are the main difference between two groups. PF, as one of the three main factors in intervention group, is mainly used to assess whether the health condition has affected the physiological activities of the patient [14]. MSAT is a therapy requires the active or passive movement cooperation of patient during the acupuncturist's manipulation at specific points. The possible mechanism of MSAT [30,31] lies in relieving muscle spasm, accelerating blood circulation and enhancing pain threshold. When the patient exercises, the mechanical conditions of the acupoints and their surroundings will change [32]. In the dynamic treatment process, from the analysis of the sensitization of the acupoints, the acupuncture points will have the effect of "force sensitivity, pressure sensitivity, and pain sensitivity" [33]. The acupuncture points are stimulated, and the curative effect is correspondingly improved. On the other side, the transmission of central motion information can inhibit the input of somatic pain information, thus MSAT can achieve a significant analgesic effect [34]. In brief, MSAT can not only improve the restricted activities of neck, but also have an analgesic effect. Therefore, MSAT has a better effect on patients with PF scores mild to moderate decline before treatment.

To patients in control group, CAT has better effect in patients scored among 56.0862 to 66.0948 in GH before treatment. GH represents an individual's evaluation of their own health status and development trends [14]. The data interval represents that patients believe that they are in good health and can maintain that level of health in the future. The number of patients with cervical spondylosis who have negative emotions is much higher than that of normal people, which may be related to chronic neck pain that affects the quality of life. Research [35] shows that patients with higher psychological scores and clear awareness of their own health status may have relatively better effects. Therefore, patients in high GH can better relief their neck pain after treatment. In other words, people with low GH score may be more suitable for other treatments, such as moxibustion, ironing, and Chinese medicine.

To sum up, patients with low role-emotional and social functioning are suitable for acupuncture treatment, among which patients with mild to moderate decline in physical functioning are more appropriate for MSAT combined with CAT, while patients with high general health may have better effect by CAT.

\section{Conclusion}

The quality of life can be comprehensively assessed before neck pain patients have relative treatment. Acupuncture can be used for patients with good emotional function and social function scores; among them, patients with good general health can be treated with conventional acupuncture therapy, and patients with mild to moderate decline in physiological function can choose motion style acupuncture therapy combined with conventional acupuncture therapy. It is hoped that through the data mining, certain ideas can be provided for the application of acupuncture clinical syndrome differentiation treatment, so as to achieve the purpose of adapting measures to individual conditions and improving curative effects.

This study is the first attempt in neck pain treatment which try to formulate related acupuncture treatments due to the objective index and subjective symptom before treatment. The selection of scale may be incomplete and due to the specialization of acupuncture manipulation, double-blind is hard to be 
performed, however assessor-blind is designed to decrease bias and the study may provide new ideas in the choice of acupuncture methods in different type of neck pain patients. Further studies can be designed to discuss indications of different acupuncture therapies, so that the clinical effect of acupuncture will be further improved.

\section{Acknowledgments}

This work was supported by the National Nation Science Foundation of China (Project no. 82074550) and the National Natural Science Foundation of Shanghai (Project no. 17ZR1430300).

\section{Author contributions}

Ruo-yun Lyu, Zong-lin Wen, Ming Gao and Bo Wang developed and designed the study. Ruo-yun Lyu and Zong-lin Wen wrote the manuscript. Wen-chao Tang, Xu-ming Yang, Jun-ling Wen and Ming Gao reviewed the manuscript. All authors read and approved the final manuscript.

\section{Conflict of interest}

None declared.

\section{Data availability}

All data generated and analyzed in the study are available from the corresponding author upon reasonable request.

\section{References}

[1] Iyer A, Azad TD, Tharin S. Cervical spondylotic myelopathy. Clin Spine Surg. 2016; 29(10): 408-414.

[2] Aljuboori Z, Boakye M. The natural history of cervical spondylotic myelopathy and ossification of the posterior longitudinal ligament: A review article. Cureus. 2019; 11(7): e5074.

[3] Hurwitz E, Randhawa K, Yu H, Côté P, Haldeman SJESJ. The Global Spine Care Initiative: A summary of the global burden of low back and neck pain studies. 2018; 27(Suppl 6): 796-801.

[4] Zheng CF, Liu YC, Hu YC, Xia Q, Miao J, Zhang JD, Zhang K. Correlations of japanese orthopaedic association scoring systems with gait parameters in patients with degenerative spinal diseases. Orthopaedic Surgery. 2016; 8(4): 447-453.

[5] Kim R, Wiest C, Clark K, Cook C, Horn MJMSP. Identifying risk factors for first-episode neck pain: A systematic review. 2018; 33: 77-83.

[6] Irnich D, Behrens N, Molzen H, Konig A, Gleditsch J, Krauss M, Natalis M, Senn E, Beyer A, Schops P. Randomised trial of acupuncture compared with conventional massage and "sham" laser acupuncture for treatment of chronic neck pain. BMJ (Clinical Research ed). 2001; 322(7302): 1574-1578.

[7] Zhang WY, Xu BF, Jin Y, Ding Y, Cai SR. Short-term and long-term effect of combination of eight confluences points on cervical spondylosis. Journal of Clinical Acupuncture and Moxibustion. 2018; 34(05): 13-17.

[8] Gao T, Zheng QH, Zhang SS, Hou TH, Li Y. Clinical application and mechanism of motion style acupuncture treatment for pain. Liaoning Journal of Traditional Chinese Medicine. 2019; 46(07): 1523-1526.

[9] Bian RM. Clinical observations on the efficacy of acupuncture kinesitherapy plus conventional acupuncture in treating cervical spondylosis. Shanghai Journal of Acupuncture and Moxibustion. 2015; 34(12): 1216-1218. 
[10] Shi GX, Liu BZ, Wang J, Fu QN, Sun SF, Liang RL, Li J, Tu JF, Tan C, Liu CZ. Motion style acupuncture therapy for shoulder pain: A randomized controlled trial. J Pain Res. 2018; 11: 2039-2050.

[11] Shi X. Acupuncture and Mobustion. Beijing: Higher Education Press; 2007.

[12] Fischer AA. Pressure algometry over normal muscles. Standard values, validity and reproducibility of pressure threshold. Pain. 1987; 30(1): 115-126.

[13] Jensen K, Andersen HO, Olesen J, Lindblom U. Pressure-pain threshold in human temporal region. Evaluation of a new pressure algometer. Pain. 1986; 25(3): 313-323.

[14] Perneger TV, Leplege A, Etter JF, Rougemont A. Validation of a French-language version of the MOS 36-Item Short Form Health Survey (SF-36) in young healthy adults. J Clin Epidemiol. 1995; 48(8): 1051-1060.

[15] Yan M, Ye F, Zhang Y, Cai X, Fu Y, Yang X. Optimization model research on efficacy in treatment of chronic urticaria by Chinese and Western medicine based on a genetic algorithm. J Tradit Chin Med. 2013; 33(1): 60-64.

[16] Liu P, Li J, Lu C, Zhao L, Lin X, Wang Y, Yang X. Data mining-based detection of the physical and chemical characteristics of Chinese medical herbs aqueous decoction in spray drying yield. Drying Technology. 2020; 1-9.

[17] Shao P, Du Z, Xin Z-P, Zheng X, Ming-lie Q, Zhu Z, Yang X-M. Electroacupuncture at Jiāj`1 (EX-B2) for lumbar intervertebral disc herniation: A randomized controlled trial. J World Journal of Acupuncture - Moxibustion. 2018.

[18] Du XZ, Bao CL, Dong GR, Yang XM. Immediate effects of scalp acupuncture with twirling reinforcing manipulation on hemiplegia following acute ischemic stroke: A hidden association study. Neural Regeneration Research. 2016; 11(5): 758-764.

[19] Yang XM, Du XZ, Dong GR. Hidden Correlation Study on the Immediate Effect of Scalp Twisting and Replenishing Therapy in the Treatment of Acute Ischemic Stroke Hemiplegia Patients. In: The 1st Huangfu Mi's Hometown Worship Ceremony and International Symposium on Academic Thoughts of Acupuncture and Moxibustion: 2012; Pingliang, Gansu, China; 2012: p. 7.

[20] Li Z. The morbility of neck sub-healthy of high school student and analysis of the risk factors. master Beijing Sport University; 2017.

[21] Meziat-Filho N, Azevedo ESG, Coutinho ES, Mendonca R, Santos V. Association between home posture habits and neck pain in High School adolescents. J Back Musculoskelet Rehabil. 2017; 30(3): 467-475.

[22] Chen J, Han JS, Fan BF, Yu SY, Wang JS. The underlying mechanisms of chronic pain: From basic to clinical. Chinese Journal of Pain Medicine. 2014; 20(02): 70-80.

[23] Shin JS, Ha IH, Lee J, Choi Y, Kim MR, Park BY, Shin BC, Lee MS. Effects of motion style acupuncture treatment in acute low back pain patients with severe disability: A multicenter, randomized, controlled, comparative effectiveness trial. Pain. 2013; 154(7): 1030-1037.

[24] Rubinstein SM, Van Tulder M. A best-evidence review of diagnostic procedures for neck and low-back pain. Best Practice \& Research Clinical Rheumatology. 2008; 22(3): 471-482.

[25] Wang Q, Bao YX. Analysis on the principle of acupuncture analgesia. China Journal of Traditional Chinese Medicine and Pharmacy. 2019; 34(10): 4911-4913.

[26] Turk DC, Wilson HD. Fear of pain as a prognostic factor in chronic pain: Conceptual models, assessment, and treatment implications. Current Pain and Headache Reports. 2010; 14(2): 88-95.

[27] Du N, Li X, Fan BF. Research progress of MAPK and NO-related signal pathways in the mechanism of electroacupuncture analgesia. Chinese Journal of Pain Medicine. 2019; 25(11): 854-858.

[28] Liang Y, Fang JQ, Du JY, Fang JF. Effect of Electroacupuncture on Activation of p38MAPK in Spinal Dorsal Horn in Rats with Complete Freund's Adjuvant-Induced Inflammatory Pain. Evid Based Complement Alternat Med. 2012; 2012: 568273.

[29] Zhang C, Xia C, Zhang X, Li W, Miao X, Zhou Q. Wrist-ankle acupuncture attenuates cancer-induced bone pain by regulating descending pain-modulating system in a rat model. Chin Med. 2020; 15: 13.

[30] Shin JS, Ha IH, Lee TG, Choi Y, Park BY, Kim MR, Lee MS. Motion style acupuncture treatment (MSAT) for acute low back pain with severe disability: A multicenter, randomized, controlled trial protocol. BMC Complement Altern Med. 2011; 11: 127

[31] Zhang Q, Yu LL, Liu SQ, Huang GY, Xu SB, Wang W. Progress of fMRI Research on "Deqi” of Acupuncture and Our Considerations About Further Studies. Zhen Ci Yan Jiu. 2018; 43(5): 330-334.

[32] Pan J, Li L, Shi WY, Chen C, Luo R, Zhong F, Yu ZA. Mechamism of moving points and acupucture rehabilition based on acupoint dynamics. Liaoning Journal of Traditional Chinese Medicine. 2019; 46(03): 618-620.

[33] Wan M, Zhou YM, Zhou J, Bao QN,Yin B, Zhang Y, Cai DJ, Liang FR. Analysis of the phenomenon and law of acupoint sensitization. Journal of Clinical Acupuncture and Moxibustion. 2017; 33(03): 74-77.

[34] Xu J. Experimental Study on the Therapeutic Effect and Mechanism of Distal Acupuncture Therapy on Cervical Spondylopathy. Hubei Minzu University; 2018.

[35] Ma C, Cao MQ, Zhou YC, Ye XJ, Yan XK. Psychological factors in correlation to acupuncture analgesia effect. Journal of Traditional Chinese Medicine. 2021; 62(01): 27-31. 